\title{
DUAL SOLUTIONS FOR HEAT AND MASS TRANSFER IN CHEMICALLY REACTING RADIATIVE NON-NEWTONIAN FLUID WITH ALIGNED MAGNETIC FIELD
}

\author{
J.V. Ramana Reddy ${ }^{1}$, V. Sugunamma ${ }^{1}$ and N. Sandeep ${ }^{2 *}$
}

${ }^{1}$ Department of Mathematics, Sri Venkateswara University, Tirupati-517502, India.

${ }^{2}$ Department of Mathematics, VIT University, Vellore-632014, India.

Email: jvr.mrkp@gmail.com, vsugunar@yahoo.co.in, dr.nsrh@gmail.com

\begin{abstract}
:
Through this paper we investigated the heat and mass transfer effects of chemically reacting radiative Casson fluid flow over a slendering/flat stretching sheet in a slip flow regime with aligned magnetic field. This study is carried out under the influence of non-uniform heat source/sink. First we converted the governing equations of the flow into ordinary differential equations by making use of suitable similarity transformations. The resultant nonlinear differential equations are solved numerically using Runge-Kutta based shooting technique. Further, graphical representations have been given to study the effects of various physical parameters on velocity, temperature and concentration fields. Also numerical computation has been carried out to investigate the influence of various physical parameters involved on skin friction, heat and mass transfer coefficients. Through this investigation, it is observed that aligned angle, Casson parameter and velocity slip parameter have tendency to control the velocity field. Also heat transfer rate in the flow over a flat stretching sheet is higher than that of slendering stretching sheet. A good agreement of the present results with the existed literature has been observed.
\end{abstract}

Keywords: Aligned magnetic field, casson fluid, slendering stretching sheet, slip flow, non-uniform heat source/sink, radiation.

\begin{tabular}{ll}
\hline NOMENCLATURE \\
$A$ & Coefficient related to stretching sheet \\
$A^{*}$ & Non-uniform heat source/sink parameter \\
$a_{1}, a_{2}$ & Constant parameters \\
$B^{*}$ & Non-uniform heat source/sink parameter \\
$B_{0}$ & Strength of the magnetic field \\
$b$ & Physical parameter related to stretching \\
& sheet \\
$C$ & Concentration of the fluid \\
$C_{f}$ & Skin-friction coefficient \\
$C_{p}$ & Specific heat at constant pressure \\
$C_{\infty}$ & Concentration far away from the sheet \\
$C_{w}(x)$ & Concentration near the sheet \\
$D_{m}$ & Mass diffusivity \\
$f_{1}$ & Maxwell's reflection coefficient \\
$h_{1}^{*}$ & Dimensional velocity slip parameter \\
$h_{2}^{*}$ & Dimensional temperature jump parameter \\
$h_{3}^{*}$ & Dimensional concentration \\
\multicolumn{3}{l}{ parameter jump } \\
$h_{1}$ & Dimensionless velocity slip parameter \\
$h_{2}$ & Dimensionless temperature jump \\
& parameter
\end{tabular}

$\begin{array}{ll}h_{3} & \begin{array}{l}\text { Dimensionless concentration jump } \\ \text { parameter }\end{array} \\ K r & \begin{array}{l}\text { Dimensionless chemical reaction } \\ \text { parameter }\end{array} \\ k & \begin{array}{l}\text { Thermal conductivity of the fluid } \\ k_{l}\end{array} \\ M & \text { Dimensional chemical reaction parameter } \\ N u_{x} & \text { Magnetic field parameter } \\ n & \text { Nusselt number } \\ \mathrm{Pr} & \text { Pelocity power index parameter } \\ \mathrm{Re}_{x} & \text { Local Reynolds number } \\ S c & \text { Schmidt number } \\ S h_{x} & \text { Sherwood number } \\ T & \text { Temperature of the fluid } \\ T_{w}(x) \quad \text { Temperature near the sheet } \\ T_{\infty} & \text { Temperature far away from the sheet } \\ u, v & \text { Velocity components in } x, y-\text { directions } \\ \text { respectively }\end{array}$

Greek symbols

$\alpha \quad$ Aligned angle

$\beta \quad$ Casson parameter

$\eta, \xi \quad$ Similarity variables

$\lambda$ Wall thickness parameter 
J. V. R. Reddy, V. Sugunamma, N. Sandeep/ Journal of Naval Architecture and Marine Engineering, 14(2017) 25-38

\section{Introduction}

Apart from water and air, most of the fluids we come across in our day to day life belong to the category of nonNewtonian fluids, which have most stability when compared with Newtonian one. So, at present most of the researchers in fluid mechanics are concentrating their research in the area of non-Newtonian fluid flows. Casson fluid is also one of the non-Newtonian fluids, which has many scientific and industrial applications. Examples of Casson fluid include paste, honey, human blood and jelly etc. The main important characteristic of Casson fluid is it exhibits the yield stress. The flow analysis over a stretching surface has many applications in engineering processes such as polymer processing, extrusion of rubber and plastic sheets. Viscous dissipation effect can alter the temperature by playing vital role as energy source. So this helps to enhance the heat transfer rate in non-Newtonian fluids. Heat and mass transfer on the free convection flow of non-Newtonian fluid past a vertical surface was discussed by Ibrahim et al. (2010). Heat transfer effects on the boundary layer flow of a non-Newtonian fluid over a stretching surface was studied by Bujurke et al. (1990). Khalid et al. (2015) analyzed the flow of Casson fluid over a vertical oscillating plate in porous medium with transverse magnetic field and found that velocity increases with an increase in phase angle but decreases with an increase in magnetic field. Raju et al. (2015) discussed the heat and mass transfer in MHD Casson fluid flow over an exponentially stretching surface. Through this study they found that heat transfer rate in Casson fluid (nonNewtonian fluid) is significantly higher than that of Newtonian fluid. The two dimensional MHD stagnation point flow of a Jeffery fluid past a heated exponentially stretching surface with convective boundary conditions was discussed by Hayat et al. (2015). The effects of Deborah and Biot numbers on Maxwell nano fluid induced by exponentially stretching sheet was studied by Mustafa et al. (2015). Khan et al. (2015) investigated the three dimensional nano fluid flows past a nonlinear stretching surface. Sandeep et al. (2015) discussed the heat transfer characteristics of an unsteady MHD dusty nano fluid flow past an exponentially stretching surface. Tripathy et al. (2015) investigated the effect of magnetic field on the flow over a moving vertical surface using convective boundary conditions.

The effect of magnetic field on Casson fluid flow over a stretching sheet with Soret and Dufour effects was analyzed by Hayat et al. (2012). Raju et al. (2015) discussed the impacts of aligned magnetic field and heat source/sink on ferrofluids over a flat plate. Sandeep and Sugunamma (2014) studied the combined effects inclined magnetic field and radiation on MHD flow past a vertical plate in porous medium and proved that inclined magnetic field slows down the motion of the fluid in both ramped and isothermal cases. The free convection flow in vertical concentric annuli under the influence of induced magnetic field was reported by Singh and Singh (2012). Dessie and Kishan (2014) discussed the impacts of variable viscosity and heat generation or absorption on MHD boundary layer flow towards a stretching surface. Paramanik (2014) discussed the effect of radiation on MHD Casson fluid flow past an exponentially stretching surface and concluded that an increase in radiation parameter causes an enhancement in the temperature of the fluid. The influence of magnetic field and radiation on unsteady flow of a rotating fluid over a stretching sheet embedded in a porous medium was investigated by Rashad (2014). Pal (2011) analyzed the effects of thermal radiation and non-uniform heat source/sink on a permeable stretching sheet. This study shows that an increase in heat absorption parameter have propensity to depreciate the temperature profiles.The effect of non-uniform source/sink on unsteady Powell-Eyring fluid flow past an inclined stretching surface was studied by Hayat et al. (2014). The influence of chemical reaction and heat source or sink on MHD stagnation point flow of a nano fluid was numerically presented by Anwar et al. (2013). Hussain et al. (2015) discussed the influence of viscous dissipation on MHD Casson nano fluid flow with convective boundary conditions. With the help of this study they found that an increase in viscous dissipation leads to an enhancement in the fluid temperature. The heat transfer analysis on the flow over an exponentially stretching surface was carried out by Mandal and Mukhopadhyay (2013). Mixed convection flow of non-Newtonian fluid over a rotating cone was discussed by Nadeem and Saleem (2014). Recently, the effect of radiation and porosity parameter on MHD three dimensional Casson fluid flow over a linearly stretching sheet was studied by Mahanta and Shaw (2015). Heat and mass transfer on an unsteady boundary layer flow over a stretching vertical slendering cylinder was reported by Patil et al. (2015). Recently, Anjali Devi and Prakash (2015) numerically discussed the MHD flow over a slendering stretching surface in a slip flow regime. In this study it is noticed that an increase in the power-law index enhances both velocity as well as temperature of the fluid. Very recently, Ali and Sandeep (2017), Mohan Krishna et al. 92016) and Sandeep (2017) analysed the heat transfer nature of MHD flows.

Still, no attempt has been made by the past researchers to investigate the heat and mass transfer in chemically reacting radiative Casson fluid flow over a slendering/flat stretching sheet in a slip flow regime with aligned magnetic field. In this study, we make an attempt to analyze these effects on Casson fluid with the help of above cited articles. Further, graphical representation has been given to study the effects of various physical 
J. V. R. Reddy, V. Sugunamma, N. Sandeep/ Journal of Naval Architecture and Marine Engineering, 14(2017) 25-38 parameters on velocity, temperature and concentration fields. Also numerical computations have been carried out to investigate the influence of various physical parameters involved in the flow on skin friction, rate of heat and mass transfer coefficients.

\section{Formulation of the Problem:}

Consider a steady, laminar, hydro magnetic two dimensional, dissipative, electrically conducting and incompressible non-Newtonian fluid flow over a slendering/flat stretching sheet in a slip flow regime. The motion of the sheet is taken along the $x$ - axis and $y$ - axis is perpendicular to the sheet. The magnetic Reynolds number is assumed to be very small such that the induced magnetic field is negligible. It is assumed that the sheet is moving with the velocity $U_{w}(x)=U_{0}(x+b)^{n}$ and the sheet is not allowing the fluid to pass through it with $v_{w}=0$. In this study $n=1$ corresponds to the flow over a flat sheet and $n \neq 1$ represents the flow over a slendering sheet. An aligned magnetic field $B(x)$ is applied to the flow along $x-$ direction as displayed in Fig.1. Thermal radiation, non-uniform heat source/sink and chemical reaction effects are taken into account.

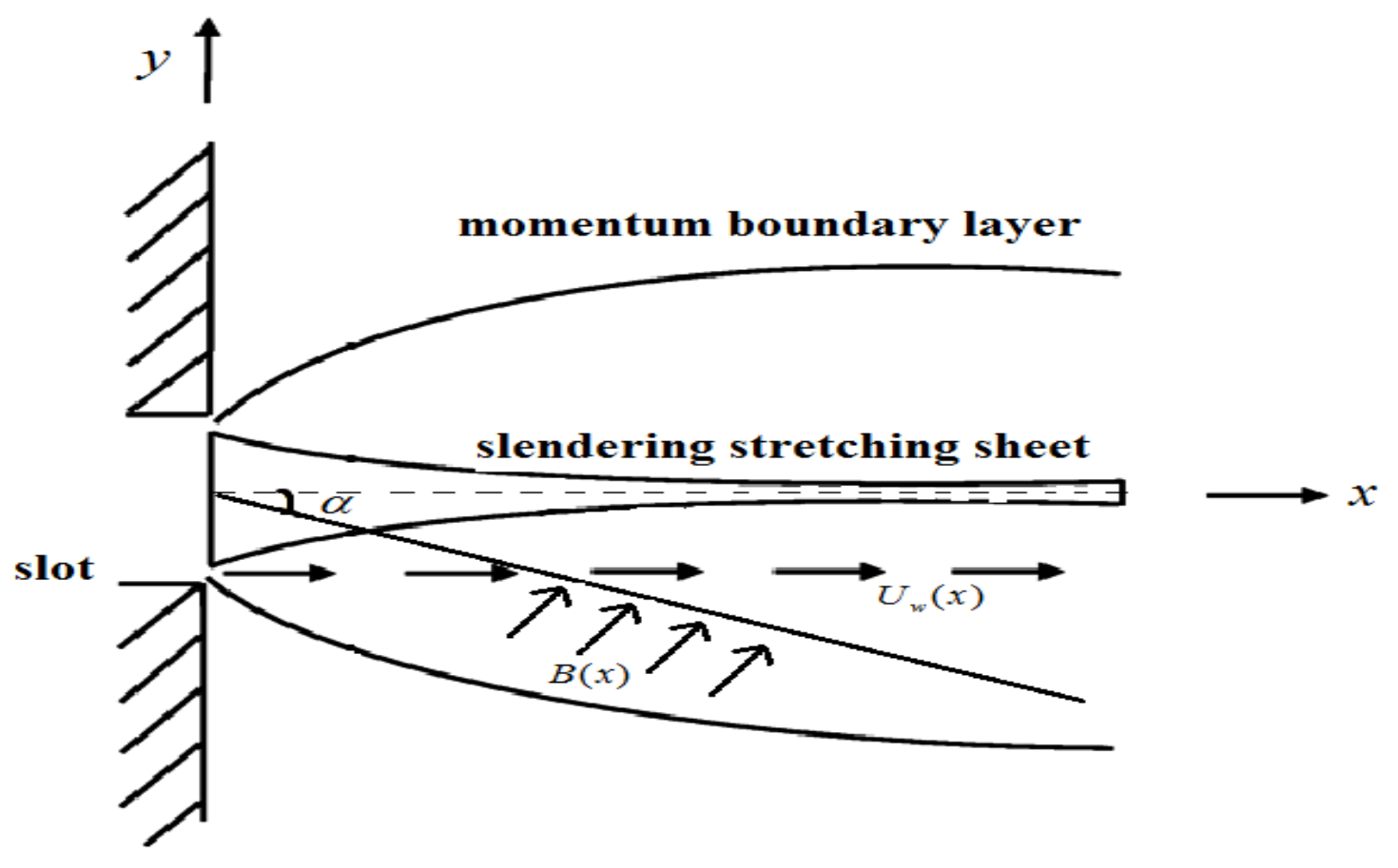

Fig.1: Physical model and coordinate system

The rheological equation of state for the Cauchy stress tensor of Casson fluid can be written as (see Paramanik (2014) and Raju (2015))

$\tau_{i j}= \begin{cases}2\left(\mu_{B}+p_{y} / \sqrt{2 \pi}\right) e_{i j}, & \pi>\pi_{c} \\ 2\left(\mu_{B}+p_{y} / \sqrt{2 \pi_{c}}\right) e_{i j}, & \pi<\pi_{c}\end{cases}$

Where $\pi=e_{i j} e_{i j}$ and $e_{i j}$ is the $(i, j)^{\text {th }}$ component of the deformation rate with itself, $\pi_{c}$ is the critical value of this product based on the non-Newtonian model, $\mu_{B}$ is the plastic dynamic viscosity of the non -Newtonian fluid and $p_{y}$ is yield stress of the fluid.

The continuity, momentum, energy and diffusion equations which describe the flow can be written as (See Anjali Devi and Prakash 2015) 
$\frac{\partial u}{\partial x}+\frac{\partial v}{\partial y}=0$

$\rho\left(u \frac{\partial u}{\partial x}+v \frac{\partial v}{\partial y}\right)=\mu\left(1+\frac{1}{\beta}\right) \frac{\partial^{2} u}{\partial y^{2}}-\sigma B(x)^{2} \sin ^{2} \alpha$,

$\rho C_{p}\left(u \frac{\partial T}{\partial x}+v \frac{\partial T}{\partial y}\right)=k \frac{\partial^{2} T}{\partial y^{2}}+\mu\left(1+\frac{1}{\beta}\right)\left(\frac{\partial u}{\partial y}\right)^{2}-\frac{\partial q_{r}}{\partial y}+q^{\prime \prime \prime}$

$u \frac{\partial C}{\partial x}+v \frac{\partial C}{\partial y}=D_{m} \frac{\partial^{2} C}{\partial y^{2}}-k_{l}\left(C-C_{\infty}\right)$,

The appropriate boundary conditions of the flow are given by,

$u\left(x, A(x+b)^{\frac{1-n}{2}}\right)=U_{w}(x)+h_{1}^{*}\left(\frac{\partial u}{\partial y}\right), v\left(x, A(x+b)^{\frac{1-n}{2}}\right)=0$,

$T\left(x, A(x+b)^{\frac{1-n}{2}}\right)=T_{w}(x)+h_{2}^{*}\left(\frac{\partial T}{\partial y}\right), C\left(x, A(x+b)^{\frac{1-n}{2}}\right)=C_{w}(x)+h_{3}^{*}\left(\frac{\partial C}{\partial y}\right)$,

$u(x, \infty)=0, T(x, \infty)=0, C(x, \infty)=0$,

in Eqn. (5),

$h_{1}^{*}=\left(\frac{2-f_{1}}{f_{1}}\right) \zeta_{1}(x+b)^{\frac{1-n}{2}}, h_{2}^{*}=\zeta_{2}\left(\frac{2-a_{1}}{a_{1}}\right)(x+b)^{\frac{1-n}{2}}, \zeta_{2}=\left(\frac{2 \gamma_{1}}{\gamma_{1}+1}\right) \frac{\zeta_{1}}{\operatorname{Pr}}$,

$h_{3}^{*}=\zeta_{3}\left(\frac{2-a_{2}}{a_{2}}\right)(x+b)^{\frac{1-n}{2}}, \zeta_{3}=\left(\frac{2 \gamma_{2}}{\gamma_{2}+1}\right) \frac{\zeta_{1}}{S c}$,

where $\rho$ is the fluid density, $u, v$ are velocity components along $x, y$-directions respectively, $\mu$ is the dynamic viscosity, $\beta=\mu_{B} \sqrt{2 \pi_{c}} / p_{y}$ is the Casson parameter, $\sigma$ is the electrical conductivity, $\alpha$ is the aligned magnetic field angle, $C_{p}$ is the specific heat capacitance, $T, C$ are temperature and concentration of the fluid near the sheet respectively, $T_{\infty}, C_{\infty}$ are temperature and concentration of the fluid in free stream, $k$ is the thermal conductivity of the fluid and $k_{l}$ is the dimensional chemical reaction parameter.

The special form for magnetic field $B(x)$, temperature $T_{w}(x)$ and concentration $C_{w}(x)$ can be taken as

$B(x)=B_{0}(x+b)^{\frac{1-n}{2}}, T_{w}(x)=T_{\infty}+T_{0}(x+b)^{\frac{1-n}{2}}, C_{w}(x)=C_{\infty}+C_{0}(x+b)^{\frac{1-n}{2}}$,

The radiative heat flux obeys the Roseland approximation $q_{r}=\frac{-4 \sigma_{e}}{3 \beta_{R}} \frac{\partial T^{4}}{\partial y}$ where $\sigma_{e}$ and $\beta_{R}$ are the Stefan-

Boltzmann constant and the coefficient of mean absorption, respectively. Fluid phase temperature differences are small enough that $T^{4}$ can be expanded in the Taylor series about temperature $T_{\infty}$ with higher order terms are neglected, then we get $T^{4} \cong 4 T_{\infty}^{3} T-3 T_{\infty}^{4}$.

The space and temperature dependent heat generation/absorption (non-uniform heat source/sink) $q^{\prime \prime \prime}$ is defined as (See Pal (2011)

$q^{\prime \prime \prime}=\left(\frac{k U_{w}(x)}{(x+b) v}\right)\left(A^{*}\left(T_{w}-T_{\infty}\right) f^{\prime}+B^{*}\left(T-T_{\infty}\right)\right)$,

where $v$ is the kinematic viscosity of the fluid, $A^{*}$ and $B^{*}$ are parameters of the space and temperature dependent internal heat generation/absorption. The positive and negative values of $A^{*}$ and $B^{*}$ represents heat generation and absorption parameters respectively

The appropriate stream function $(\psi)$ and similarity transformations are 
$\left.\begin{array}{l}\psi(x, y)=f(\xi) \sqrt{\left(\frac{2}{1+n}\right) v U_{0}(x+b)^{1+n}}, \xi=y \sqrt{\left(\frac{1+n}{2}\right) \frac{U_{0}(x+b)^{n-1}}{v}}, \\ \theta=\frac{T-T_{\infty}}{T_{w}(x)-T_{\infty}}, \phi=\frac{C-C_{\infty}}{C_{w}(x)-C_{\infty}},\end{array}\right\}$

The velocity components $u, v$ in $x, y$ - directions are defined in terms of stream function as follows $u=\partial \psi / \partial y, v=-\partial \psi / \partial x$.

Using Eqn. (9), we get

$u=U_{0}(x+b)^{n} f^{\prime}, v=-\sqrt{\left(\frac{1+n}{2}\right) v U_{0}(x+b)^{n-1}}\left(f-\xi f^{\prime}\left(\frac{1-n}{1+n}\right)\right)$,

Now, substitution of Eqn. (10) automatically satisfies the continuity Eqn. (1). Further using Eqs. (8)-(10) in Eqs. (2)-(4) gives,

$$
\begin{aligned}
& \left(1+\frac{1}{\beta}\right) f^{\prime \prime \prime}-\left(\frac{2 n}{n+1}\right) f^{\prime 2}+f f^{\prime \prime}-\left(\frac{2}{n+1}\right) M \sin ^{2} \alpha f^{\prime}=0, \\
& \left(1+\frac{4}{3} R\right) \theta^{\prime \prime}-\operatorname{Pr}\left(\left(\frac{1-n}{1+n}\right) f^{\prime} \theta-f \theta^{\prime}\right)+E c\left(1+\frac{1}{\beta}\right)\left(f^{\prime \prime}\right)^{2}+\left(\frac{2}{1+n}\right)\left(A^{*} f^{\prime}+B^{*} \theta\right)=0, \\
& \phi^{\prime \prime}-S c\left(\left(\frac{1-n}{1+n}\right) f^{\prime} \phi-f \phi^{\prime}\right)-K r S c \phi=0,
\end{aligned}
$$

The corresponding boundary conditions will be transformed as

$$
\begin{aligned}
& f(\lambda)=\lambda\left(\frac{1-n}{1+n}\right)\left(1+h_{1} f^{\prime \prime}(0)\right), f^{\prime}(\lambda)=1+h_{1} f^{\prime \prime}(0), \\
& \theta(\lambda)=1+h_{2} \theta^{\prime}(0), \phi(\lambda)=1+h_{3} \phi^{\prime}(0), \\
& f^{\prime}(\infty)=0, \theta(\infty)=0, \phi(\infty)=0,
\end{aligned}
$$

where $\lambda=A \sqrt{\frac{(1+n) U_{0}}{2 v}}, h_{1}=\sqrt{\frac{(1+n) U_{0}}{2 v}}\left(\frac{2-f_{1}}{f_{1}}\right) \zeta_{1}$,

$h_{2}=\sqrt{\frac{(1+n) U_{0}}{2 v}}\left(\frac{2-a_{1}}{a_{1}}\right) \zeta_{2}, h_{3}=\sqrt{\frac{(1+n) U_{0}}{2 v}}\left(\frac{2-a_{2}}{a_{2}}\right) \zeta_{3}$,

Here in the above Eqs. (11)-(14), the prime indicates differentiation with respect to $\xi$. Also, $M=\frac{\sigma B_{0}^{2}}{\rho U_{0}}$, $\operatorname{Pr}=\frac{\mu C_{p}}{k}, E c=\frac{U_{w}^{2}}{C_{p} T_{0}}, R=\frac{4 \sigma_{e} T_{\infty}^{3}}{k \beta_{R}}, S c=\frac{v}{D}$, and $K r=\frac{k_{l}(x+b)^{1-n}}{U_{0}}$ are magnetic field parameter, Prandtl number, Eckert number, radiation parameter, Schmidt number and chemical reaction parameter respectively. Eqs.(11)-(13) along with the boundary conditions in Eqn. (14) are non-linear differential equations with the domain $[\lambda, \infty)$. In order to facilitate the computations in the domain into $[0, \infty)$, we define a new function $F$ as $F(\eta)=F(\xi-\lambda)=f(\xi)$.

Therefore the Eqs. (11)-(14) become,

$$
\begin{aligned}
& \left(1+\frac{1}{\beta}\right) F^{\prime \prime \prime}-\left(\frac{2 n}{n+1}\right) F^{\prime 2}+F F^{\prime \prime}-\left(\frac{2}{n+1}\right) M \sin ^{2} \alpha F^{\prime}=0, \\
& (1+R) \Theta^{\prime \prime}-\operatorname{Pr}\left(\left(\frac{1-n}{1+n}\right) F^{\prime}-F \Theta^{\prime}\right)+E c\left(1+\frac{1}{\beta}\right)\left(F^{\prime \prime}\right)^{2}+\left(\frac{2}{1+n}\right)\left(A^{*} F^{\prime}+B^{*} \Theta\right)=0, \\
& \Phi^{\prime \prime}-S c\left(\left(\frac{1-n}{1+n}\right) F^{\prime} \Phi-F \Phi^{\prime}\right)-K r S c \Phi=0,
\end{aligned}
$$

with the corresponding boundary conditions 


$$
\begin{aligned}
& F(0)=\lambda\left(\frac{1-n}{1+n}\right)\left(1+h_{1} F^{\prime \prime}(0)\right), F^{\prime}(0)=1+h_{1} F^{\prime \prime}(0), \\
& \Theta(0)=1+h_{2} \Theta^{\prime}(0), \Phi(\lambda)=1+h_{3} \Phi^{\prime}(0), \\
& F^{\prime}(\infty)=0, \Theta(\infty)=0, \Phi(\infty)=0,
\end{aligned}
$$

The physical quantities in view of engineering applications are Skin-friction coefficient $\left(C_{f}\right)$, Nusselt number $\left(N u_{x}\right)$ and Sherwood number $\left(S h_{x}\right)$, which are calculated as given below.

The Skin-friction coefficient $\left(C_{f}\right)$ is defined as

$C_{f}=\frac{2 \tau_{w}}{\rho U_{w}^{2}}$, where the local shear stress $\left(\tau_{w}\right)$ is given by

$\tau_{w}=\mu\left(\frac{\partial u}{\partial y}\right)_{y=A(x+b)^{\frac{1-n}{2}}}$

$C_{f}\left(\operatorname{Re}_{x}\right)^{\frac{1}{2}}=\left(1+\frac{1}{\beta}\right) \sqrt{2(n+1)} F^{\prime \prime}(0)$,

Nusselt number is given by

$N u_{x}=\frac{(x+b) q_{w}}{k\left(T_{w}(x)-T_{\infty}\right)}$, where the heat flux at the surface $\left(q_{w}\right)$ is given by

$q_{w}=-k\left(\frac{\partial T}{\partial y}\right)_{y=A(x+b)^{\frac{1-n}{2}}}$

$N u_{x}\left(\operatorname{Re}_{x}\right)^{\frac{-1}{2}}=-\sqrt{\frac{n+1}{2}} \Theta^{\prime}(0)$,

Finally, the Sherwood number is defined as

$S h_{x}=\frac{(x+b) q_{m}}{D_{m}\left(C_{w}(x)-C_{\infty}\right)}$, where the mass flux at the surface $\left(q_{m}\right)$ is given by

$q_{m}=-D_{m}\left(\frac{\partial C}{\partial y}\right)_{y=A(x+b)^{\frac{1-n}{2}}}$,

$S h_{x}\left(\operatorname{Re}_{x}\right)^{\frac{-1}{2}}=-\sqrt{\frac{n+1}{2}} \Phi^{\prime}(0)$,

where $\operatorname{Re}_{x}=\frac{(x+b) U_{w}(x)}{v}$ is the local Reynolds number.

\section{Results and Discussion}

In order to explore the effects of various physical parameters on the flow, heat and mass transfer, the system of nonlinear ordinary differential Eqs. (15)- (17) along with the boundary conditions Eqn. (18) are solved numerically by making use of Runge-Kutta based shooting technique. Further, the effects of various physical parameters namely, aligned magnetic field angle $(\alpha)$, Casson parameter $(\beta)$, radiation parameter $(R)$, Eckert number $(E c)$, heat source /sink parameters $\left(A^{*}, B^{*}\right)$, chemical reaction parameter $(K r)$, velocity slip parameter $\left(h_{1}\right)$, temperature jump parameter $\left(h_{2}\right)$ and concentration parameter $\left(h_{3}\right)$ have been discussed through graphs. Also tabular forms are given to study the effects of these parameters on friction factor $\left(C_{f}\right)$, local Nusselt ( $N u_{x}$ ) and Sherwood $\left(S h_{x}\right)$ numbers.

For numerical results we considered $\beta=2, \quad M=3, \alpha=\pi / 3, R=2, \operatorname{Pr}=4, A^{*}=B^{*}=0.1, \quad K r=\lambda=0.5$, $S c=1, h_{1}=h_{2}=h_{3}=0.5$.These values have been kept in common throughout our analysis except the varied values are shown in respective figures and tables. All the results have been discussed for both flat stretching sheet $(n=1)$ and slendering stretching sheet $(n=0.5)$ cases. 


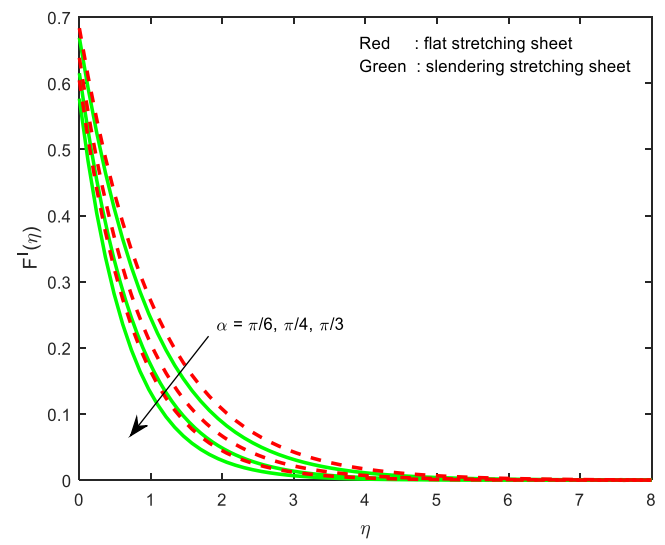

Fig. 2: Effect of aligned angle $(\alpha)$ on velocity profiles

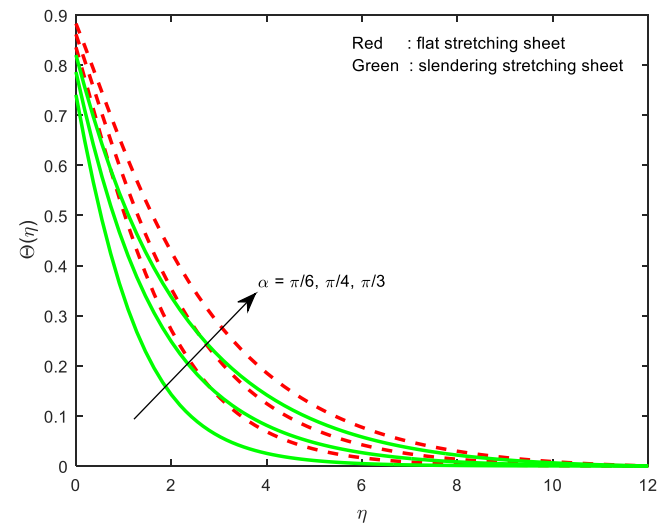

Fig. 3: Effect of aligned angle $(\alpha)$ on temperature profiles

Figs. 2-4 depict the effect of aligned angle $(\alpha)$ on velocity, temperature and concentration profiles. It can be observed from Fig. 1 that an increase in aligned angle depreciates the velocity profiles. This may happen due to the fact that an increase in aligned angle of the magnetic field strengthens the applied magnetic field, which produces a resistive type of force in the flow called Lorentz force. This force works in the opposite direction of the fluid motion and restricts the fluid velocity. From Figs. 3 and 4 one can conclude that an increase in aligned angle leads to an enhancement in both temperature as well as concentration profiles. As the Lorentz force works as agent to generate the heat in the flow, an increase in aligned angle improves the thickness of thermal and concentration boundary layers.

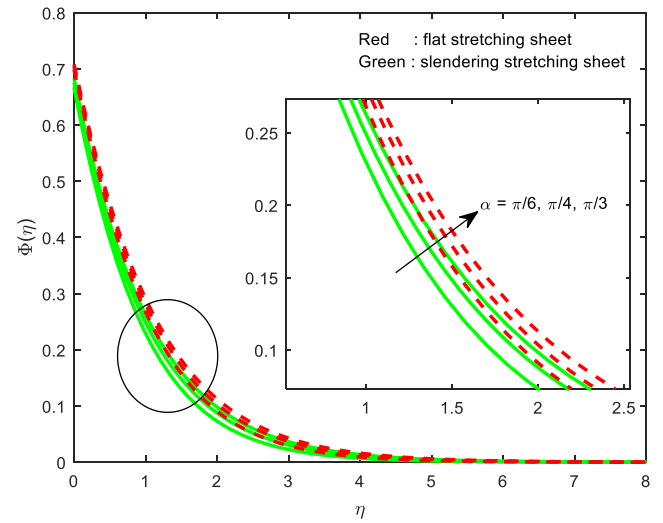

Fig. 4: Effect of aligned angle $(\alpha)$ on concentration profiles

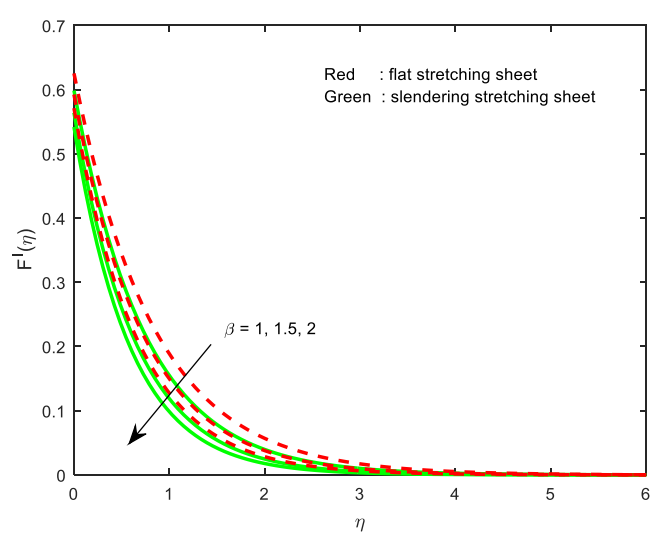

Fig. 5: Effect of Casson parameter $(\beta)$ on velocity profiles

Figs. 5-7 reveal the effect of Casson parameter $(\beta)$ on the flow. It is found that an increase in Casson parameter suppresses the fluid velocity. This may happen due to the decreasing nature of momentum boundary layer thickness with increasing values of Casson parameter. Physically, the stress at the yield point will decrese with an increase in Casson parameter. But reverse action takes place in case of temperature and concentration distribution as shown in Figs. 6 and 7 respectively. This is because of increase in Casson parameter helps to enhance the thermal as well as concentration boundary layer thicknesses.

Fig. 8 portrays the temperature distribution for different values of radiation parameter $(R)$. It is evident that an increase in radiation parameter enhances the fluid temperature. Generally, an increase in radiation generates the heat energy in the flow and this leads to an increase in the temperature of the fluid. Fig. 9 exhibits the temperature profiles for distinct values of Eckert number $(E c)$. It is clear that an increase in the Eckert number enhances the temperature profiles. This may happen due to the fact that increase in dissipation develops friction in the flow. As a result heat will be generated in the flow. So we observe thicker thermal boundry layer with increasing values of $E c$. 


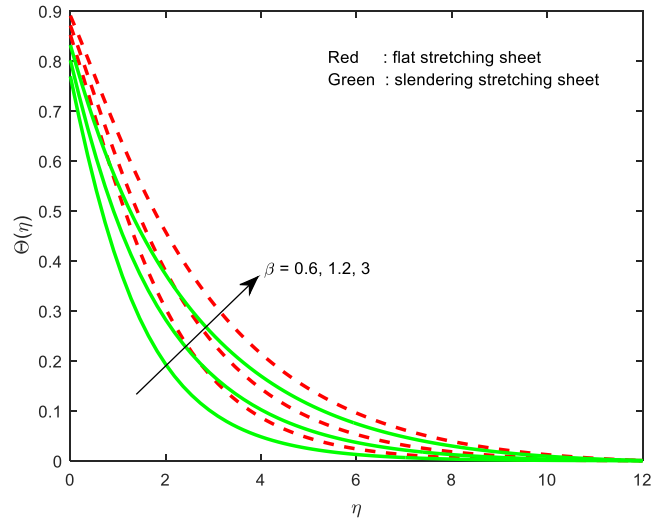

Fig. 6: Effect of Casson parameter $(\beta)$ on temperature profiles

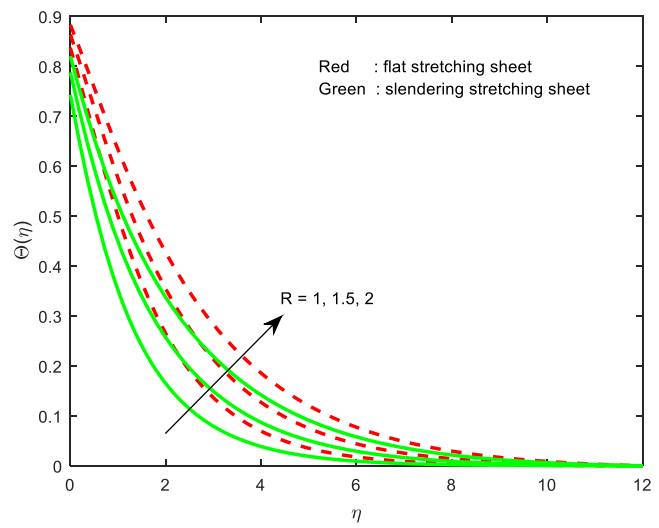

Fig. 8: Effect of radiation $(R)$ on temperature profiles

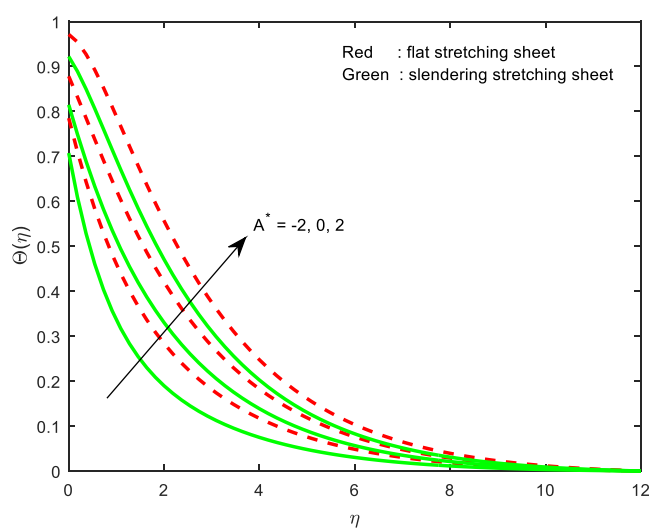

Fig. 10: Effect of non-uniform heat source/sink parameter $\left(A^{*}\right)$ on temperature profiles

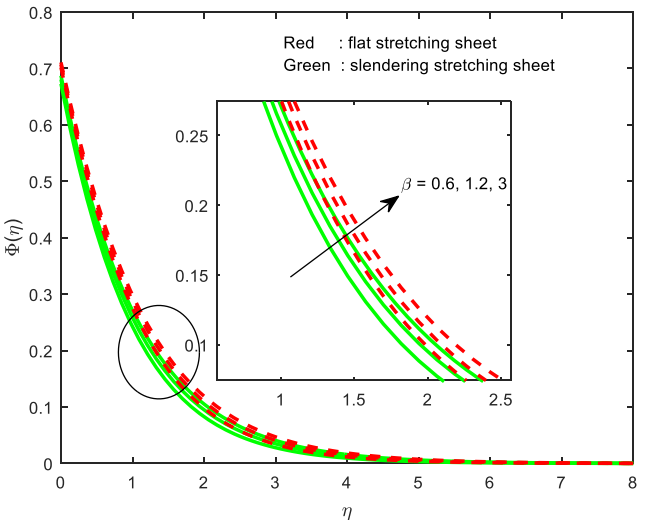

Fig. 7: Effect of Casson parameter $(\beta)$ on concentration profiles

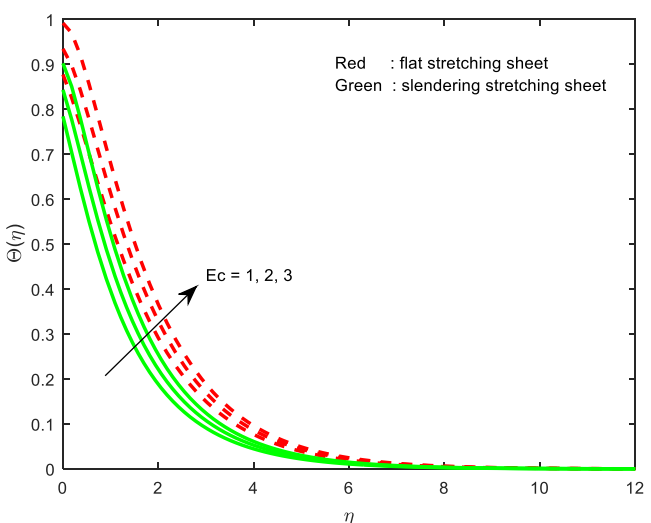

Fig. 9: Effect of Eckert number ( $E c$ ) on temperature profiles

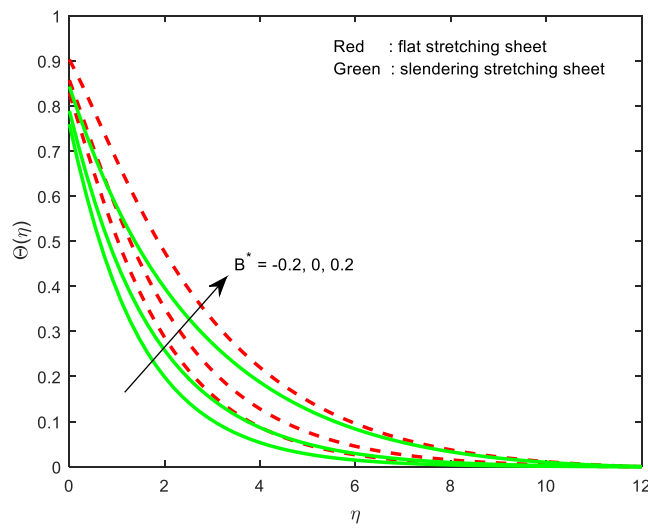

Fig. 11: Effect of non-uniform heat source/sink parameter $\left(B^{*}\right)$ on temperature profiles

Figs. 10 and 11 reveal the effect of non-uniform heat source/sink parameters $\left(A^{*}, B^{*}\right)$ on temperature field. We can say that increase in non-uniform heat source/sink parameters $A^{*}$ and $B^{*}$ leads to a raise in the temperature of the fluid. Generally, the positive values of $A^{*}$ and $B^{*}$ generate the heat and this leads to an enhancement in the thermal boundary layer thickness and negative values of $A^{*}$ and $B^{*}$ absorb the heat, which causes for decrease in the thermal boundary layer thickness. 


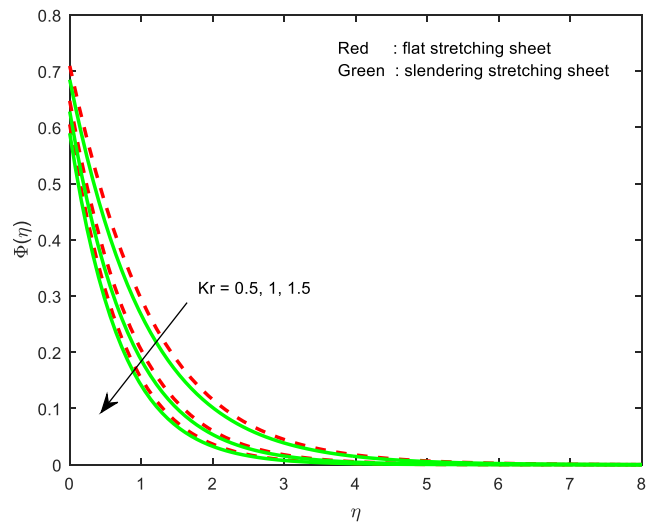

Fig. 12: Effect of chemical reaction parameter $(\mathrm{Kr})$ on concentration profiles

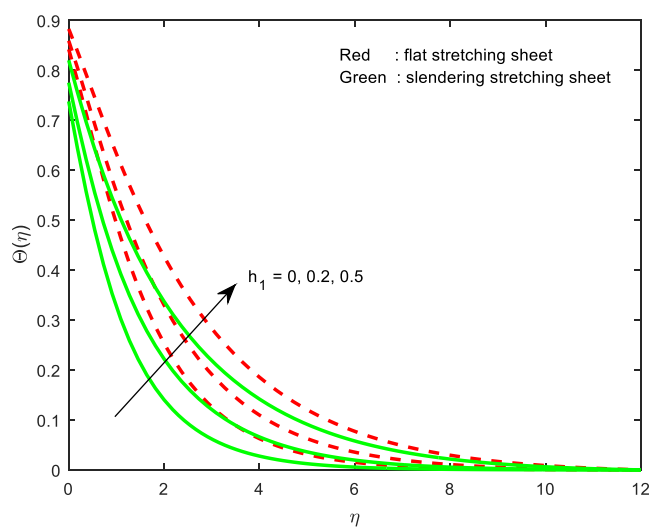

Fig. 14: Effect of velocity slip parameter $\left(h_{1}\right)$ on temperature profiles

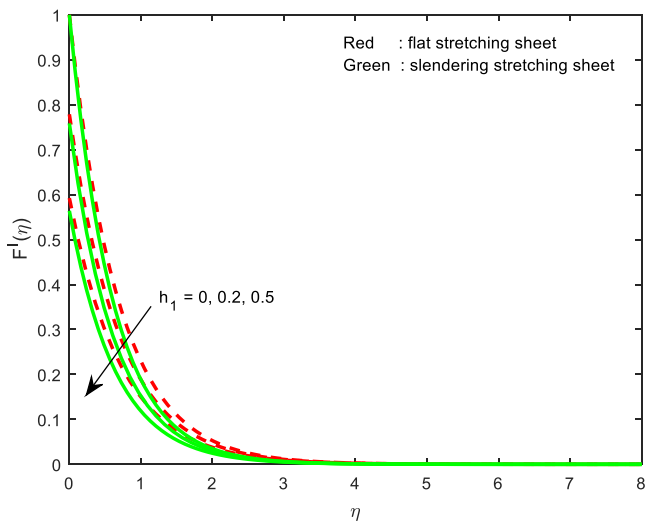

Fig. 13: Effect of velocity slip parameter $\left(h_{1}\right)$ on velocity profiles

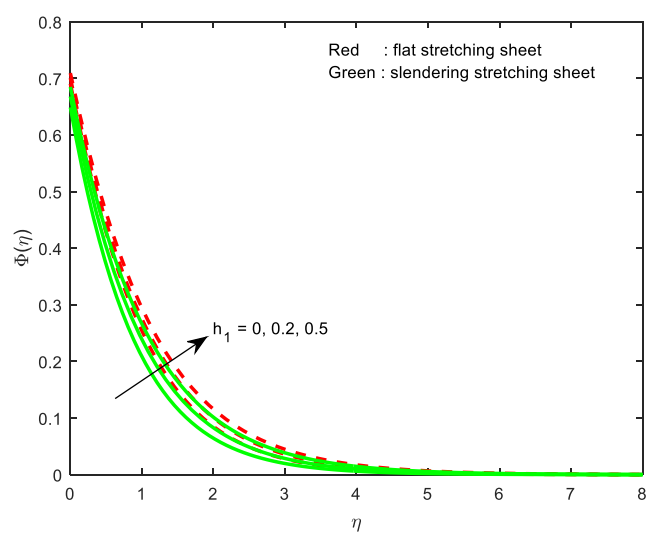

Fig. 15: Effect of velocity slip parameter $\left(h_{1}\right)$ on concentration profiles

Fig. 12 depicts the influence of chemical reaction parameter $(K r)$ on concentration profiles. It is easy to examine that increasing values of chemical reaction parameter depreciates the concentration field. This is due to the increase in the rate of interfacial mass transfer. Figs. 13-15 display the impact of dimensionless slip velocity parameter $\left(h_{1}\right)$ on the flow. Fig. 13, it is found that an increase in the values of velocity slip parameter reduces the velocity profiles. Because not all the pulling force of the stretching sheet can be transformed to the fluid, but it gets increased away from the sheet. Hence slip velocity exerts a progressively diminishing effect leading to the reversed effect.

Figs. 14 and 15 respectively highlight the effect of velocity slip parameter on temperature and concentration profiles respectively. An increase in velocity slip parameter helps for heat generation in the flow. So the velocity slip parameter has the tendency to increase the thicknesses of the thermal and concentration boundary layers. Therefore we observe a hike in both temperature and concentration profiles.

Fig. 16 reveals the effect of temperature jump parameter $\left(h_{2}\right)$ on temperature profiles. It is found that an increase in the temperature jump parameter decreases the temperature profiles. This is due to the fact that an increase in the temperature jump parameter enhances the thermal accommodation coefficient and this leads to depreciation in the thermal diffusion towards the flow. The effect of concentration parameter $\left(h_{3}\right)$ on concentration distribution was elucidated in Fig. 17. It is evident that an increase in concentration jump parameter reduces the concentration profiles.

Table 1 shows the comparison of the present results with the existed results of Khader and Megahed (2013). We found an excellent agreement with the existed results under some special conditions. This shows the validity of the present work and the numerical technique we used in this study. 


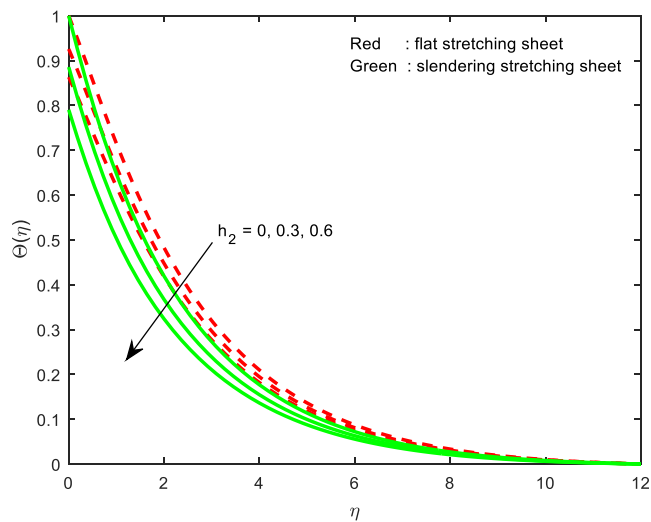

Fig. 16: Effect of temperature jump parameter $\left(h_{2}\right)$ on temperature profiles

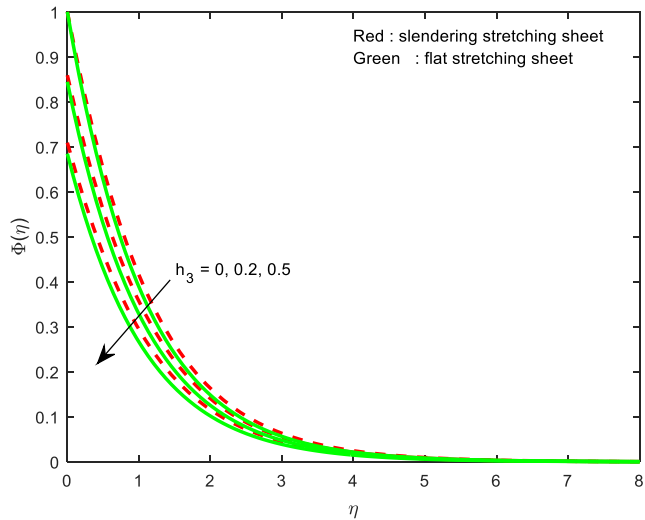

Fig. 17: Effect of concentration jump parameter $\left(h_{3}\right)$ on concentration profiles

Table 1: Comparison of the values of $F^{\prime \prime}(0)$ when $R=\alpha=M=h_{2}=h_{3}=A^{*}=B^{*}=E c=S c=0, n=0.5$

\begin{tabular}{|cccc|}
\hline$\lambda$ & $h_{1}$ & $\begin{array}{c}\text { Khader and Megahed } \\
(2013) \\
F^{\prime \prime}(0)\end{array}$ & $\begin{array}{c}\text { Present study } \\
F^{\prime \prime}(0)\end{array}$ \\
\hline 0.2 & 0 & -0.924828 & -0.924821 \\
\hline 0.25 & 0.2 & -0.733395 & -0.7333941 \\
\hline 0.5 & 0.2 & -0.759570 & -0.759570 \\
\hline
\end{tabular}

Tables 2 and 3 give the numerical computations to study the effects of various physical parameters on skinfriction coefficient, local Nusselt and Sherwood numbers for both cases, viz. i) flow past a flat stretching sheet ( $n=1)$ and ii) flow past a slendering stretching sheet $(n=0.5)$ respectively. It is evident from Tables $2 \& 3$ that increasing values of aligned angle of the magnetic filed and Casson parameter depreciate the friction factor along with the heat and mass transfer coefficients. Radiation and dissipation parameters have tendency to reduce the heat transfer rate while chemical reaction parameter have proclivity to enhance the mass transfer rate. Velocity slip, temperature and concentration jump parameters have tendency to reduce the heat and mass transfer rates.

Table 2: Variation in skin friction, heat and mass transfer coefficients for the flow over a flat stretching sheet ( $n=1)$.

\begin{tabular}{|c|c|c|c|c|c|c|c|c|c|c|c|}
\hline$\alpha$ & $\beta$ & $R$ & $E c$ & $A^{*}$ & $K r$ & $h_{1}$ & $h_{2}$ & $h_{3}$ & $F^{\prime \prime}(0)$ & $-\Theta^{\prime}(0)$ & $-\Phi^{\prime}(0)$ \\
\hline$\pi / 6$ & & & & & & & & & -0.6549 & 0.3290 & 0.6004 \\
\hline$\pi / 4$ & & & & & & & & & -0.7463 & 0.2772 & 0.5893 \\
\hline$\pi / 3$ & & & & & & & & & -0.8153 & 0.2342 & 0.5815 \\
\hline & 0.6 & & & & & & & & -0.6859 & 0.2963 & 0.5965 \\
\hline & 1.2 & & & & & & & & -0.7692 & 0.2582 & 0.5866 \\
\hline & 3.0 & & & & & & & & -0.8427 & 0.2192 & 0.5785 \\
\hline & & 1.0 & & & & & & & -0.8153 & 0.3259 & 0.5815 \\
\hline & & 1.5 & & & & & & & -0.8153 & 0.2729 & 0.5815 \\
\hline & & & 1 & & & & & & -0.8153 & 0.2457 & 0.5815 \\
\hline & & & 2 & & & & & & -0.8153 & 0.1313 & 0.5815 \\
\hline
\end{tabular}


J. V. R. Reddy, V. Sugunamma, N. Sandeep/Journal of Naval Architecture and Marine Engineering, 14(2017) 25-38

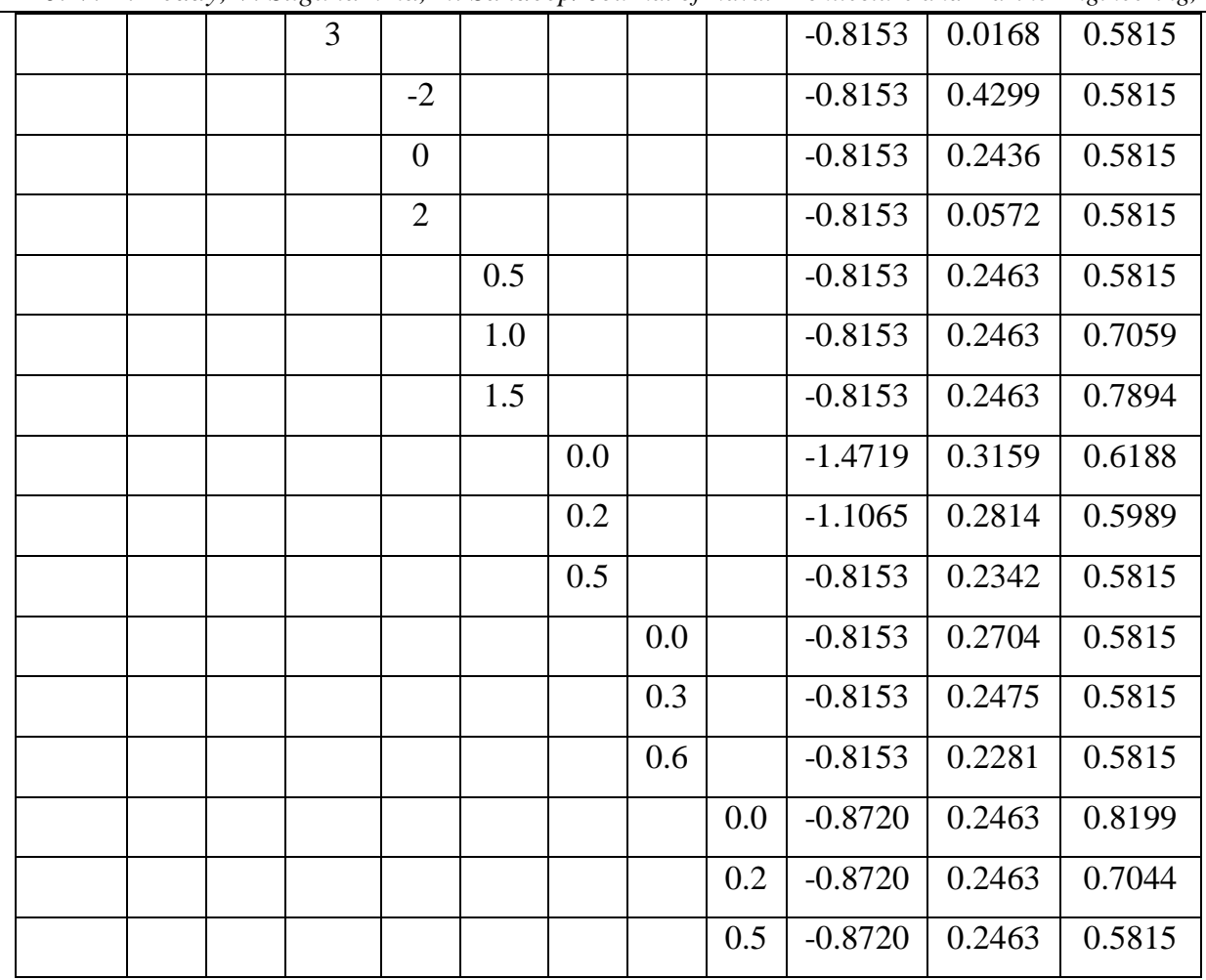

Table 3: Variation in skin friction, heat and mass transfer coefficients for the flow over a slendering stretching sheet $(n=0.5)$.

\begin{tabular}{|c|c|c|c|c|c|c|c|c|c|c|c|}
\hline$\alpha$ & $\beta$ & $R$ & $E c$ & $A^{*}$ & $K r$ & $h_{1}$ & $h_{2}$ & $h_{3}$ & $F^{\prime \prime}(0)$ & $-\Theta^{\prime}(0)$ & $-\Phi^{\prime}(0)$ \\
\hline$\pi / 6$ & & & & & & & & & -0.6852 & 0.5187 & 0.6634 \\
\hline$\pi / 4$ & & & & & & & & & -0.7942 & 0.4275 & 0.6439 \\
\hline \multirow[t]{15}{*}{$\pi / 3$} & & & & & & & & & -0.8720 & 0.3597 & 0.6305 \\
\hline & 0.6 & & & & & & & & -0.7359 & 0.4620 & 0.6544 \\
\hline & 1.2 & & & & & & & & -0.8237 & 0.3973 & 0.6388 \\
\hline & 3.0 & & & & & & & & -0.9006 & 0.3366 & 0.6257 \\
\hline & & 1.0 & & & & & & & -0.8720 & 0.5145 & 0.6305 \\
\hline & & 1.5 & & & & & & & -0.8720 & 0.4245 & 0.6305 \\
\hline & & 2.0 & & & & & & & -0.8720 & 0.3597 & 0.6305 \\
\hline & & & 1 & & & & & & -0.8720 & 0.4318 & 0.6305 \\
\hline & & & 2 & & & & & & -0.8720 & 0.3138 & 0.6305 \\
\hline & & & 3 & & & & & & -0.8720 & 0.1957 & 0.6305 \\
\hline & & & & -2 & & & & & -0.8720 & 0.5852 & 0.6305 \\
\hline & & & & 0 & & & & & -0.8720 & 0.3704 & 0.6305 \\
\hline & & & & 2 & & & & & -0.8720 & 0.1556 & 0.6305 \\
\hline & & & & & 0.5 & & & & -0.8720 & 0.3703 & 0.6305 \\
\hline & & & & & 1.0 & & & & -0.8720 & 0.3703 & 0.7437 \\
\hline
\end{tabular}




\begin{tabular}{|l|l|l|l|l|l|l|l|l|l|l|l|}
\hline & & & & & 1.5 & & & & -0.8720 & 0.3703 & 0.8209 \\
\hline & & & & & & 0.0 & & & -1.6445 & 0.5243 & 0.7026 \\
\hline & & & & & & 0.2 & & & -1.2066 & 0.4492 & 0.6639 \\
\hline & & & & & & 0.5 & & & -0.8720 & 0.3597 & 0.6305 \\
\hline & & & & & & & 0.0 & & -0.8720 & 0.4202 & 0.6305 \\
\hline & & & & & & & 0.3 & & -0.8720 & 0.3816 & 0.6305 \\
\hline & & & & & & & 0.6 & & -0.8720 & 0.3496 & 0.6305 \\
\hline & & & & & & & & 0.0 & -0.8720 & 0.3703 & 0.9208 \\
\hline & & & & & & & & 0.2 & -0.8720 & 0.3703 & 0.7776 \\
\hline & & & & & & & & 0.5 & -0.8720 & 0.3703 & 0.6305 \\
\hline
\end{tabular}

\section{Conclusions}

The present paper deals with the study of heat and mass transfer effects on Casson fluid flow over a slendering/flat stretching sheet. The influence of various physical parameters on the flow was shown through graphs and tabular forms. The conclusions of the present study are mentioned below.

- Velocity profiles were depreciated by the influence of Casson parameter and velocity slip parameter.

- Aligned angle, Casson parameter, velocity slip parameter, Eckert number, heat source/sink parameters and radiation parameter have tendency to enhance the fluid temperature.

- It is interesting to note that flow through flat stretching sheet shows better heat transfer performance while compared with the flow past a slendering stretching sheet.

- Increase in either aligned angle or Casson parameter leads to a decrease in friction factor along with heat and mass transfer coefficients.

- Aligned magnetic field have tendency to control the momentum boundary layer thickness.

\section{References}

Ali, M.E., and Sandeep, N. (2017): Cattaneo-Christov model for radiative heat transfer of magnetohydrodynamic Casson-ferrofluid: a numerical study, Results in Physics, Vol. 7, pp.21-30.

https://doi.org/10.1016/j.rinp.2016.11.055

Anjalidevi, S. P., and Prakash, J. (2015): Temperature dependent viscosity and thermal conductivity effects on hydromagnetic flow over a slendering stretching sheet, Journal of the Nigerian Mathematical Society, Vol. 34, No.3, pp. 318-330. https://doi.org/10.1016/j.jnnms.2015.07.002

Anwar, I., Kasim, A. R. M., Ismail, Z., Salleh, M. Z. and Shafie, S. (2013): Chemical reaction and uniform heat generation or absorption effects on mhd stagnation-point flow of a nanofluid over a porous sheet, World Applied Sciences Journal, Vol. 24, No.10, pp. 1390-1398.

http://dx.doi.org/10.5829/idosi.wasj.2013.24.10.1307

Bujurke, N. M., Biradar, S. N. and Hiremath, P. S. (2015): Heat transfer in the boundary layer of non-newtonian fluid flow past a stretching sheet, Proceedings of Indian National Science Academy, Vol. 56, No.4, pp. 353-360.

Dessie, H. and Kishan, N. (2014): MHD effects on heat transfer over stretching sheet embedded in porous medium with variable viscosity, Viscous Dissipation and Heat Source/Sink, Ain Shams Enineering Journal, Vol. 5, No.5, pp. 967-977. https://doi.org/10.1016/j.asej.2014.03.008

Hayat, T., Asad, S., Mustafa, M. and Alsaedi, A. (2015): MHD stagnation point flow of jeffrey fluid over a convectively heated stretching sheet, Computers and Fluids, Vol. 108, pp. 179-185. https://doi.org/10.1016/j.compfluid.2014.11.016 
J. V. R. Reddy, V. Sugunamma, N. Sandeep/Journal of Naval Architecture and Marine Engineering, 14(2017) 25-38

Hayat, T., Asad, S., Mustafa, M. and Alsaedi, A. (2014): Radiation effects on the flow of powell-eyring fluid past an unsteady inclined stretching sheet with non uniform heat source/sink, PLoS ONE, Vol. 9, No.7, e103214. https://doi.org/10.1371/journal.pone.0103214

Hayat, T., Shehzad, S. A. and Alsaedi, A. (2012): Soret and Dufour effects on magnetohydrodynamic (MHD) flow of casson fluid, Applied Mathematics and Mechanics (English Edition), Vol. 33, No.10, pp. 1301-1312. http://dx.doi.org/10.1007/s10483-012-1623-6

Hussain, T., Shehzad, S. A., Alsaedi. A., Hayat, T. and Ramzan, M. (2015): Flow of casson nanofluid with viscous dissipation and convective conditions: a mathematical model, Journal of Central South University, Vol. 22, No.3, pp. 1132-1140. http://dx.doi.org/10.1007/s11771-015-2625-4

Ibrahim, F. S., Hady, F. M., Abdel-Gaied., S. M. and Eid M. R. (2010): Influence of chemical reaction on heat and mass transfer of non-newtonian fluid with yield stress by free convection from vertical surface in porous medium considering Soret effect, Applied Mathematics and Mechanics, Vol. 31, No.6, pp. 675-684. http://dx.doi.org/10.1007/s10483-010-1302-9

Khader, M., and Megahed A. M. (2013): Numerical solution for boundary layer flow due to a nonlinearly stretching sheet with variable thickness and slip velocity, The European Physical Journal Plus, Vol. 128, No.100, pp.100-108. http://dx.doi.org/10.1140/epjp/i2013-13100-7

Khalid, A., Khan, A. and Shafie S. (2015): Unsteady MHD free convection flow of casson fluid past over an oscillating vertical plate embedded in a porous medium, Engineering Science and Technology, an International Journal, Vol. 18, No.3, pp.309-317. https://doi.org/10.1016/i.jestch.2014.12.006

Khan, J. A., Mustafa, M., Hayat, T. and Alsaedi, A. (2015): Three dimensional flow of nanofluid over a nonlinearly stretching sheet: an application to solar energy, International Journal of Heat and Mass Transfer Vol. 86, pp. 158-164. https://doi.org/10.1016/j.ijheatmasstransfer.2015.02.078

Krishna, M. P., Sandeep, N., Reddy, J.V.R., Sugunamma, V. (2016): Dual solutions for unsteady flow of Powell-Eyring fluid past an inclined stretching sheet, Journal of Naval Architechure and Marine Engineering, 13, 89-99, 2016.http://dx.doi.org/10.3329/jname.v13i1.25338

Mahanta, G. and Shaw, S. (2015): 3D casson fluid flow past a porous linearly stretching sheet with convective boundary condition, Alexandria Engineering Journal, Vol. 54, No.3, pp. 653-659. https://doi.org/10.1016/j.aej.2015.04.014

Mandal, I. C. and Mukhopadhyay, S. (2013): Heat transfer analysis for fluid flow over an exponentially stretching porous sheet with surface heat flux in porous medium, Ain Shams Engineering Journal, Vol. 4, No.1, pp. 103-110. https://doi.org/10.1016/j.asej.2012.06.004

Mustafa, M., Khan, J. A., Hayat, T. and Alsaedi, A. (2015): Simulations for Maxwell fluid flow past a convectively heated exponentially stretching sheet with nanoparticles, AIP Advances, Vol. 5, No.3, 037133. http://dx.doi.org/10.1063/1.4916364

Nadeem, S. and Saleem, S. (2014): Mixed convection flow of Eyring-Powell fluid along a rotating cone, Results in Physics, Vol. 4, pp. 54-62. https://doi.org/10.1016/j.rinp.2014.03.004

Pal, D. (2011): Combined effects of non-uniform heat source/sink and thermal radiation on heat transfer over an unsteady stretching permeable surface, Communications in Nonlinear Science and Numerical Simlation, Vol. 16, No.4, pp. 1890-1904. https://doi.org/10.1016/j.cnsns.2010.08.023

Paramanik, S. (2014): Casson fluid flow and heat transfer past an exponentially porous stretching surface in presence of thermal radiation, Ain Shams Engineering Journal, Vol. 5, No.1, pp. 205-212. https://doi.org/10.1016/j.asej.2013.05.003

Patil, P. M., Roy, S., and Pop, I.(2013): Chemical reaction effects on unsteady mixed convection boundary layer flow past a permeable slender vertical cylinder due to nonlinearly stretching velocity, Chemical Engineering Communications, Vol. 200, No.3, pp. 398-417.

http://dx.doi.org/10.1080/00986445.2012.712578

Raju, C. S. K., Sandeep, N., Sugunamma, V., Babu, M. J. and Ramana Reddy, J. V. (2016): Heat and mass transfer in magneto hydrodynamic casson fluid over an exponentially permeable stretching surface, Engineering Science and Technology an International Journal, Vol. 19, No. 1, pp. 45-52.

https://doi.org/10.1016/j.jestch.2015.05.010

Raju, C. S. K., Sandeep, N., Sulochana C. and Sugunamma, V. (2015): Effects of aligned magnetic field and radiation on the flow of ferrofluids over a flat plate with non-uniform heat source/sink, International Journal of Science and Engineering, Vol. 8, No.2, pp. 151-158. 
J. V. R. Reddy, V. Sugunamma, N. Sandeep/Journal of Naval Architecture and Marine Engineering, 14(2017) 25-38 http://dx.doi.org/10.12777/ijse.8.2.151-158

Rashad, A. M. (2014): Effects of radiation and variable viscosity on unsteady MHD flow of a rotating fluid from stretching surface in porous medium, Journal of Egyptian Mathematical Society, Vol. 22, No.1, pp. 134-142. https://doi.org/10.1016/j.joems.2013.05.008

Sandeep, N. and Sugunamma, V. (2014): Radiation and inclined magnetic field effects on unsteady hydro magnetic free convection flow past an impulsively moving vertical plate in a porous medium, Journal of Applied Fluid Mechanics, Vol. 7, No.2, pp. 275-286.

Sandeep, N., Sulochana, C. and Kumar, B. R. (2016): Unsteady MHD radiative flow and heat transfer of dusty nanofluid over an exponentially stretching surface, Engineering Science and Technology an International Journal, Vol. 19, No. 1, 227-240. https://doi.org/10.1016/j.jestch.2015.06.004

Sandeep, N. (2017): Effect of aligned magnetic field on liquid thin film flow of magnetic-nanofluids embedded with graphene nanoparticles, Advanced Powder Technology, Vol.28, No.3, pp. 865-875.

https://doi.org/10.1016/j.apt.2016.12.012

Singh, R. K. and Singh, A. K. (2012): Effect of induced magnetic field on natural convection in vertical concentric annuli, Acta Mechanica Sinica, Vol. 28, No.2, pp. 315-323.

https://doi.org/10.1007/s10409-012-0052-4

Tripathy, R. S., Dash, G. C., Mishra, S. R. and Bagg, S. (2015): Chemical reaction effect on MHD free convective surface over a moving vertical plate through porous medium, Alexandria Engineering Journal, Vol. 54, No.3, pp. 673-679. https://doi.org/10.1016/j.aej.2015.04.012 